\title{
Interleukin- 6 and activin A are independently associated with cardiovascular events and mortality in type 2 diabetes: the prospective Asker and Bærum Cardiovascular Diabetes (ABCD) cohort study
}

Anne Pernille Ofstad ${ }^{1 *}$, Lars Gullestad ${ }^{2,3}$, Elsa Orvik ${ }^{1}$ Svend Aakhus², Knut Endresen², Thor Ueland ${ }^{4}$, Pål Aukrust ${ }^{4,5,6}$, Morten W Fagerland ${ }^{7}$, Kåre I Birkeland ${ }^{8}$ and Odd Erik Johansen ${ }^{1}$

\begin{abstract}
Background: Novel and robust cardiovascular (CV) markers are needed to improve CV morbidity and mortality risk prediction in type 2 diabetes (T2D). We assessed the long term predictive value of 4 novel CV risk markers for major $\mathrm{CV}$ events and mortality.

Methods: We included patients with T2D who had cytokines (interleukin [IL]-6 and activin A [actA]), a maximum stress ECG test (evaluated by the normalization pattern in early recovery phase) and echocardiography (evaluated by a measure of the left ventricular filling pressure - E/Em) assessed at baseline. The primary endpoint was time to first of any of the following events: myocardial infarction, stroke, hospitalization for unstable angina pectoris and death. All outcomes were adjudicated by independent experts. We used Cox proportional hazard modeling, Harrell C-statistic and the net reclassification improvement (NRI) to assess the additional value beyond conventional markers (age, gender, prior CV disease, HDL, creatinine, diastolic BP, microalbuminuria).

Results: At baseline the study cohort ( $n=135$, mean age/diabetes duration/HbA1c: $59 \mathrm{yrs} / 7 \mathrm{yrs} / 7.6 \%$ [59 mmol/mol], 26\% females) had moderate elevated CV risk (42\% microalbuminuria, mean Framingham 10 year CV-risk 9.6\%). During $8.6 \mathrm{yrs} / 1153.7$ person years, 26 patients experienced 36 events. All 4 novel risk markers were significantly associated with increased risk of the primary endpoint, however, only IL-6 and actA improved C-statistic and NRI (+0.119/43.2\%, +0.065 / $20.3 \%$ respectively) compared with the conventional $\mathrm{CV}$ risk factors.
\end{abstract}

Conclusions: IL- 6 and actA may provide prognostic information on CV events and mortality in T2D beyond conventional CV risk factors.

Trial registration: ClinicalTrials.gov: NCT00133718

Keywords: Type 2 diabetes, Cardiovascular event, MACE, Inflammatory marker, Cardiovascular risk, Risk prediction

\footnotetext{
* Correspondence: Annepernille@hotmail.com

'Department of Medical Research, Bærum Hospital, Vestre Viken Hospital

Trust, N-1309 Rud, Bærum, Norway

Full list of author information is available at the end of the article
} 


\section{Introduction}

Individuals with type 2 diabetes are at high risk for developing cardiovascular disease (CVD), such as coronary artery disease $(\mathrm{CAD})$ and stroke, and CVD is recognized as the leading cause of premature death in people with type 2 diabetes [1]. Although state-of-the-art follow-up, including primary and secondary prevention, has reduced the cardiovascular $(\mathrm{CV})$ incidence in individuals with type 2 diabetes, considerable residual $\mathrm{CV}$ risk remains. Therefore, together with increasing longevity, the CV burden for societies remains substantial [2]. One particular challenge in diabetes is that detection of CVD, in particular of CAD, is difficult due to atypical symptoms as well as a low sensitivity inherent in traditional non-invasive tests such as exercise ECG test or scintigraphy [3]. Moreover, although certain biomarkers have held the promise to improve CV risk prediction in people with type 2 diabetes, their applicability and implementation currently are not widespread. Novel, preferable, non-invasive tests (functional or structural) and markers (blood or urine) are therefore needed to enable earlier identification of individuals with type 2 diabetes at high risk for CVD.

Exercise ECG is frequently used to diagnose CAD in subjects with type 2 diabetes, however with lower sensitivity than in non-diabetic subjects. An evaluation of the early recovery phase of an ECG stress-test may potentially provide insight into the interplay between physiology and pathology $[4,5]$ and our group has previously shown that detection of a pathological normalization of this phase improves sensitivity of an ECG stress test [6]. Based on these data, we hypothesized that detection of a pathological recovery phase would improve prediction of adverse outcome in people with type 2 diabetes. Another functional parameter, readily available from a conventional echocardiography examination, is the ratio $\mathrm{E} / \mathrm{Em}$ which describes the ratio between early diastolic transmitral flow velocity and early diastolic mitral annular velocity. $\mathrm{E} / \mathrm{Em}$ is a sensitive, non-invasive measure of the left ventricular end diastolic filling pressure in selected populations [7] and has been found to be independently associated with mortality and $\mathrm{CV}$ events in several disease condititions [8-10]. Since patients with type 2 diabetes are prone to develop left ventricular diastolic dysfunction, which often evolves over several years before symptoms occur [11], we hypothezised that $\mathrm{E} / \mathrm{Em}$ could provide prognostic information also in this population.

In addition to novel approaches in ECG- and echocardiography assessment, soluble biomarkers have received much attention in CVD [12-15]. A large mendelian randomization analysis [15] found evidence for a causal role of IL-6 receptor signalling in the development of $\mathrm{CAD}$, and proposed blockage of the IL- 6 receptor as a novel therapeutic approach to prevent CAD. Our group has previously shown that interleukin (IL)- 6 and activin A, a member of the transforming growth factor $\beta$ superfamily, are related to the degree of CAD in patients with type 2 diabetes $[16,17]$.

In the present study, in individuals with type 2 diabetes, we explored the association of these novel CV risk markers (pathological recovery phase, E/Em, IL-6 and activin A) with major $\mathrm{CV}$ events and mortality.

\section{Research design and methods}

\section{Study population and study design}

The present longitudinal, cohort study is part of the prospective study Asker and Bærum Cardiovascular Diabetes (ABCD) study (clinical trials.gov id: NCT00133718) that was initiated in 2002. In short, 135 patients with type 2 diabetes were recruited over 2 years (2002-2004) and underwent a comprehensive diagnostic work-up at baseline (BL) including medical history and physical examination, laboratory assessment including markers of inflammation, renal function and lipids, urinary assessment of albumin excretion, ECG, cardiopulmonary exercise test (CPET) with special emphasis on the recovery phase (ST/HR-recovery loop evaluation), echocardiography including tissue Doppler Imaging (TDI), and invasive coronary angiography.

Our primary composite endpoint was time to death or first CV event (myocardial infarction [MI], stroke or hospitalization for unstable angina pectoris [UAP]). All clinical events were adjudicated by an independent endpoint committee. During the first two years of the study, 120 of the 135 patients participated in a two year program comparing usual care provided by general practitioners to intensive structured care at the diabetic out-patient clinic. At the end of the 2 year program, all study participants were transferred back to pre-inclusion care and recommended follow-up according to guidelines.

Informed consent was obtained from all participants. The study was conducted in accordance with the Helsinki declaration and good clinical practice and was approved by the Regional Ethical Committee.

\section{Biochemical analyses in blood}

Routine laboratory parameters were assessed in peripheral venous blood drawn in the morning after an overnight fast and immediately analyzed by the local laboratory (described in details previously [16]). Plasma levels of IL- 6 and serum levels of activin A were analyzed by immunoassays from R\&D Systems (Minneapolis, Minn., USA) and Serotec (Oxford, UK) as previously described [16,17]. Serum levels of high sensitivity C-reactive protein (hsCRP) were analyzed by a high-sensitivity, particleenhanced, immunoturbidimetric assay on a modulator platform (Roche Diagnostica, Basel, Switzerland). For all assays, the intra-and interassay coefficients of variation were $<10 \%$. 


\section{Urinary samples}

Urinary albumin excretion was determined in timed overnights urine samples. Micro-albuminuria was defined as urinary excretion $>20 \mu \mathrm{g} / \mathrm{min}$ in two out of three samples [18].

\section{Exercise testing}

Exercise capacity was assessed by a modified conventional maximum symptom-limited cardiopulmonary exercise test on a cycle ergometer as described previously [16]. The ST/ heart rate (HR)-slope was computer calculated using linear regression of all ST-amplitude and HR-data (obtained every $10^{\text {th }}$ second) during the last 4 minutes of exercise. The computer generated ST/HR- recovery loop was then evaluated in the same lead and semi-quantitatively classified to be counterclockwise (normal) or clockwise or figure 8 loop (pathological).

\section{Coronary angiography}

Coronary angiography was performed in 91 patients according to standard procedures irrespective of results from non-invasive tests [16]. Significant CAD was defined as the presence of $\geq 50 \%$ luminal diameter narrowing of one or more of the epicardial arteries or its major branches.

\section{Echocardiography}

Transthoracic echocardiography was obtained in 100 consenting patients by experienced research echocardiographers following guidelines for acquisition and interpretation of data $[19,20]$ using GE Vingmed Ultrasound's Vivid 5 or 7 with a $2.5 \mathrm{MHz}$ probe (Horten, Norway). Pulsed wave Doppler mitral flow velocities were recorded in the apical 4-chamber view with the sample volume between the leaflet tips. In the same view, color-coded Tissue Doppler Imaging (TDI) loops were recorded and stored digitally and the early diastolic mitral annular velocity (Em) was measured off-line in the basal interventricular septum (EchoPac, GE Vingmed Ultrasound AS).

\section{Statistics}

Based on the STENO-2 study ( 715 patient years of follow-up) [21], we targeted at least 1000 patient years of follow-up to ensure that the predictive properties of the novel risk factors and tests could be assessed with a reasonable power. We used the two-sample T test to compare continuous variables. Microalbuminuria, activin $\mathrm{A}$ and hsCRP were log-transformed due to their skewed distributions. The Pearson chi-square test and the Fisher mid-p test were used to compare dichotomous variables [22].

We explored the relation of biomarkers to outcome with Cox proportional hazards models. The following variables were considered for inclusion in adjusted models: age, diabetes duration, gender, known history of CVD i.e.,
$\mathrm{CAD}$, stroke or peripheral artery disease, at inclusion, allocation to treatment group first 2 study years (see methods), systolic blood pressure (BP), diastolic BP, HDL cholesterol, LDL cholesterol, HbA1c, creatinine, body mass index (BMI), smoking, microalbuminuria, left ventricle internal diastolic diameter, end diastolic volume index, and LV ejection fraction (LVEF)). Variables with $\mathrm{p}>0.25$ in univariable analyses were excluded from the final adjusted models. We assessed the proportional hazards assumption by a test based on Schoenfeld's residuals.

To explore the additional predictive value of the new risk markers, we used Harrell's C-statistic and the Net Reclassification Improvement (NRI) [23]. Three risk categories (defined by $5 \%$ and $10 \%$ cut-offs of estimated probabilities from logistic regression models) were used to calculate the NRIvalues. We used SPSS version 18.0 (SPSS Inc., Chicago, IL) and STATA version 12 (StataCorp, College Station, TX) for statistical analysis. In all analyses, $\mathrm{p}<0.05$ was considered significant.

\section{Results}

The observational period, which started in 2002, ended May $29^{\text {th }} 2012$ and of the 135 patients that were enrolled and comprehensively assessed at BL, three patients $(2.2 \%)$ were lost to follow-up, rendering 132 patients with information on outcome and vital status at study end. In two cases, cause of death was lacking in the medical records, and information was obtained from the Norwegian National Death Registry. During a follow-up time of $8.6 \pm 2.1$ years corresponding to 1153 person years, 26 patients (19.3\%) experienced a total of 36 events ( 8 MIs, 8 strokes, 3 hospitalizations for UAP, $5 \mathrm{CV}$ deaths and 12 non-CV deaths [3 cancers, 2 infections, 1 Parkinson's Disease, 1 acute renal failure, 1 opioid overdose and 1 gangrenous colon due to embolies]) translating to an event rate of 3.12 and a crude mortality rate of 1.47 pr 100 person years.

Baseline characteristics stratified by outcome are given in Table 1 and Figure 1A-E. Patients who experienced an event were significantly older, had higher proportions of 2or 3-vessel CAD, had more often known CAD or CVD at inclusion, had higher serum level of creatinine, lower LVEF and a higher Framingham risk score. In addition, these patients more often had a pathological recovery-loop as well as higher levels of IL- 6 , activin A and E/Em. In contrast, there were no differences in hsCRP, lipid parameters, HbA1c and microalbuminuria between these two groups of type 2 diabetes patients (Table 1, Figure 1A-E).

In a univariate Cox regression analysis, IL-6, activin A, $\mathrm{E} / \mathrm{Em}$ and pathological recovery-loop, but not CRP (data not shown), were all significantly associated with the outcome (Figure 2). Several conventional risk factors were also significantly associated with outcome in univariate analysis: age $(\mathrm{HR}=1.08,95 \% \mathrm{CI} 1.03-1.13)$, known history 
Table 1 Demographics, traditional CV risk factors, comorbidities and the use of medication at baseline, stratified by outcome

\begin{tabular}{|c|c|c|c|c|}
\hline & All $(n=135)$ & Patients with event $(n=26)$ & Patients without event $(n=109)$ & $P$ value \\
\hline \multicolumn{5}{|l|}{ Conventional CV risk markers } \\
\hline Age (yrs) & $58.5 \pm 10.0$ & $64.2 \pm 8.6$ & $57.2 \pm 9.9$ & 0.001 \\
\hline Females n (\%) & $35(26)$ & $29(27)$ & $6(23)$ & 0.81 \\
\hline Diabetes duration (yrs) & $6.5 \pm 6.3$ & $5.6 \pm 4.8$ & $6.6 \pm 6.4$ & 0.46 \\
\hline $\mathrm{BMI}\left(\mathrm{kg} / \mathrm{m}^{2}\right)$ & $30.0 \pm 5.4$ & $29.7 \pm 6.2$ & $30.1 \pm 5.2$ & 0.75 \\
\hline Systolic BP (mmHg) & $142 \pm 20$ & $144 \pm 21$ & $142 \pm 20$ & 0.60 \\
\hline Diastolic BP (mmHg) & $83 \pm 10$ & $83 \pm 13$ & $83 \pm 9$ & 0.81 \\
\hline $\mathrm{HbA1c}(\% / \mathrm{mmol} / \mathrm{mol})$ & $7.6 \pm 1.6 / 59.3 \pm 17.0$ & $7.5 \pm 1.6 / 58.3 \pm 17.0$ & $7.6 \pm 1.6 / 59.6 \pm 17.1$ & 0.74 \\
\hline $\mathrm{FBG}(\mathrm{mmol} / \mathrm{L})$ & $9.5 \pm 3.2$ & $9.4 \pm 3.2$ & $9.6 \pm 3.3$ & 0.80 \\
\hline Total cholesterol (mmol/L) & $4.9 \pm 1.0$ & $4.8 \pm 1.4$ & $5.0 \pm 1.0$ & 0.47 \\
\hline LDL cholesterol (mmol/L) & $2.8 \pm 0.9$ & $2.7 \pm 0.8$ & $2.9 \pm 0.9$ & 0.23 \\
\hline $\mathrm{HDL}$ cholesterol (mmol/L) & $1.3 \pm 0.4$ & $1.2 \pm 0.3$ & $1.3 \pm 0.4$ & 0.29 \\
\hline Triglycerides (mmol/L) & $1.9 \pm 1.8$ & $2.2 \pm 3.2$ & $1.8 \pm 1.2$ & 0.56 \\
\hline Creatinine $(\mu \mathrm{mol} / \mathrm{L})$ & $78.5 \pm 15.3$ & $86.7 \pm 21.1$ & $76.5 \pm 12.9$ & 0.025 \\
\hline Albuminuria ( $\mu \mathrm{g} / \mathrm{min})$ & $16.0(9.0,30.5)$ & $22.0(9.8,39.0)$ & $15.0(9.0,28.3)$ & 0.091 \\
\hline Microalbuminuria n (\%) & $56(42)$ & $14(54)$ & $42(39)$ & 0.17 \\
\hline LVEF (\%) & $63 \pm 8$ & $59 \pm 8$ & $64 \pm 8$ & 0.029 \\
\hline Daily smoking n (\%) & $16(12)$ & $3(12)$ & $13(12)$ & 0.87 \\
\hline Known CAD at inclusion n (\%) & $16(12)$ & $8(31)$ & $8(7)$ & 0.002 \\
\hline Known CVD at inclusion $n(\%)^{*}$ & $22(16)$ & $10(39)$ & $12(11)$ & 0.001 \\
\hline Framingham 10 years risk of CHD (\%) & $9.6 \pm 6.6$ & $12.7 \pm 6.9$ & $8.9 \pm 6.4$ & 0.022 \\
\hline \multicolumn{5}{|l|}{ Medications } \\
\hline Numbers of Blood glucose lowering medications & $1.2 \pm 0.9$ & $1.4 \pm 0.9$ & $1.2 \pm 0.9$ & 0.29 \\
\hline Numbers of BP-lowering medicationst & $0.9 \pm 1.0$ & $1.1 \pm 1.2$ & $0.9 \pm 1.0$ & 0.39 \\
\hline On statins n (\%) & $61(45)$ & $14(54)$ & $47(50)$ & 0.38 \\
\hline On ASA n (\%) & $45(33)$ & $12(46)$ & $33(30)$ & 0.16 \\
\hline
\end{tabular}

Data given as means $\pm S D$, medians (interquartile range) or proportions.

Abbreviations: CV Cardiovascular, BMI Body mass index, BP Blood Pressure, FBG Fasting blood glucose, $L V E F$ Left ventricular ejection fraction, CAD Coronary artery disease, CVD Cardiovascular disease, CHD Coronary heart disease, ACEl Angiotensin-converting enzyme inhibitor, ARB Angiotensin II receptor blocker, ASA Acetylsalicylic acid.

* = known history of CAD, stroke or peripheral artery disease at inclusion.

$\dagger=\mathrm{ACEl} / \mathrm{ARBs} /$ beta blockers/Ca-blockers/diuretics/combined therapy.

of CVD at inclusion $(\mathrm{HR}=3.53,95 \% \mathrm{CI} 1.58-7.87)$ and creatinine ( $\mathrm{HR}=1.41,95 \%$ CI 1.09-1.82).

Activin A and IL-6 (also when dichotomized at median values, respectively IL- $6>0.6 \mathrm{pg} / \mathrm{mL}$ and activin $\mathrm{A}>0.56$ $\mathrm{ng} / \mathrm{mL}$ ), but not $\mathrm{E} / \mathrm{Em}$ and pathological recovery loop maintained their predictive value when adjusted for the conventional risk factors (age, gender, known CV disease, diastolic blood pressure, microalbuminuria and serum levels of HDL-cholesterol and creatinine) in multivariate analysis (Figure 2). Table 2 shows C-statistics and NRI for our conventional risk factors (clinical and biochemical) and different combinations of novel biomarkers on top of this standard model. Two of the explored novel markers (i.e., IL-6 and activin A) significantly improved risk classification in this study cohort and fitted overall with the results from the Cox regression. Moreover, when combining these two markers, we observed a $47 \%$ improvement in risk classification.

\section{Discussion}

Our main findings in the present study were that the novel risk markers and tests IL-6, activin A, pathological recovery loop and $\mathrm{E} / \mathrm{Em}$ all were significantly associated with a combined endpoint of $\mathrm{CV}$ events and mortality in a cohort of patients with type 2 diabetes at moderate $\mathrm{CV}$ risk. For IL-6 and activin A, the association to CV events and mortality was kept when adjusting for traditional CV risk factors. Also, when added to a model based on traditional risk factors, IL-6 and activin A, but not E/Em or postexercise recovery loop, improved risk classification beyond conventional CV risk factors as assessed by $\mathrm{C}$-statistics and NRI. 

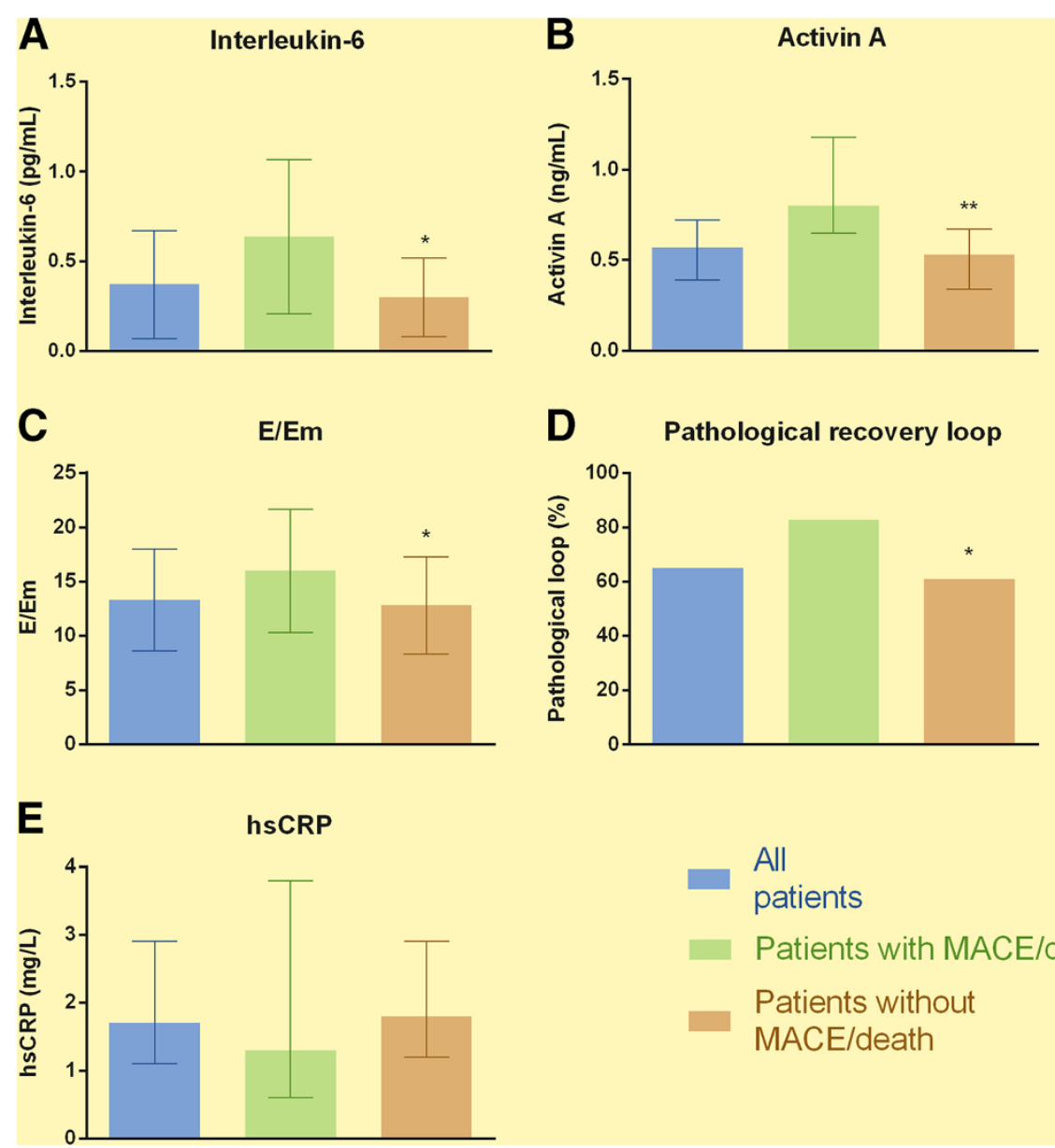

Figure 1 A-E: Novel cardiovascular risk markers stratified by outcome. The columns represent the mean \pm SD [IL-6 and E/Em] or median (IQ range) [activin A and hsCRP] levels of novel CV markers, or proportions of participants with pathological loop [pathological loop] in all participants, in those who experienced a MACE or died, and in those who stayed event-free. $*: p<0.05, * *: p<0.01$ event vs no event. SD = standard deviation, IL-6 = Interleukin-6, hSCRP = high sensitivity C-reactive protein, CV = cardiovascular, MACE = major adverse cardiovascular event.

Of interest is that IL-6 has emerged as an important cytokine with a link to atherosclerosis and diabetes [24,25], and evidence from two large genetic studies indicates causality between IL-6 receptor signalling and CAD [15,26]. Supportive to our results are the results by Martin-Cordero et al. of increased levels of IL-6 in high CV risk rats (genetically obese rats with metabolic syndrome) [27], and the study by Zhou et al. which found increased levels of IL-6 in epicardial tissue in humans with CAD [28], highlighting this association. Further, longitudinal studies in healthy post-menopausal women [29], healthy men [30] and elderly [31] have shown the ability of IL- 6 to be a predictor of future CV events and mortality. However, data evaluating IL6's long term ability to predict clinical outcomes in people with type 2 diabetes is scarce. In a relatively small crosssectional study on men with type 2 diabetes, comparison of different $\mathrm{CV}$ risk markers in patients with microvascular complications with patients with established CAD [32], revealed no significant difference in IL-6 levels. A German group investigated the association between IL- 6 and primary CV events in 1072 patients with type 2 diabetes during 5 years follow-up and found a significant association with adverse events [33]. Our study further indicates an association between IL- 6 and CV events and all cause mortality in people with type 2 diabetes potentially giving prognostic information beyond traditional risk factors.

Activin A exhibits both anti- and pro inflammatory effects [34] and has been implicated in the pathogenesis of diabetes. It has also been related to CAD, potentially reflecting a counteracting mechanism [35], and may reflect disease severity of type 2 diabetes $[17,36,37]$. The present study further supports a role for activin A in diabetes and related $\mathrm{CV}$ events and death by showing an independent association between activin $\mathrm{A}$ and adverse outcome in type 2 diabetes.

CRP is regarded as a reliable down-stream marker of inflammation, and although IL-6 is suggested to be one of its main inducers, we could not find any association of 


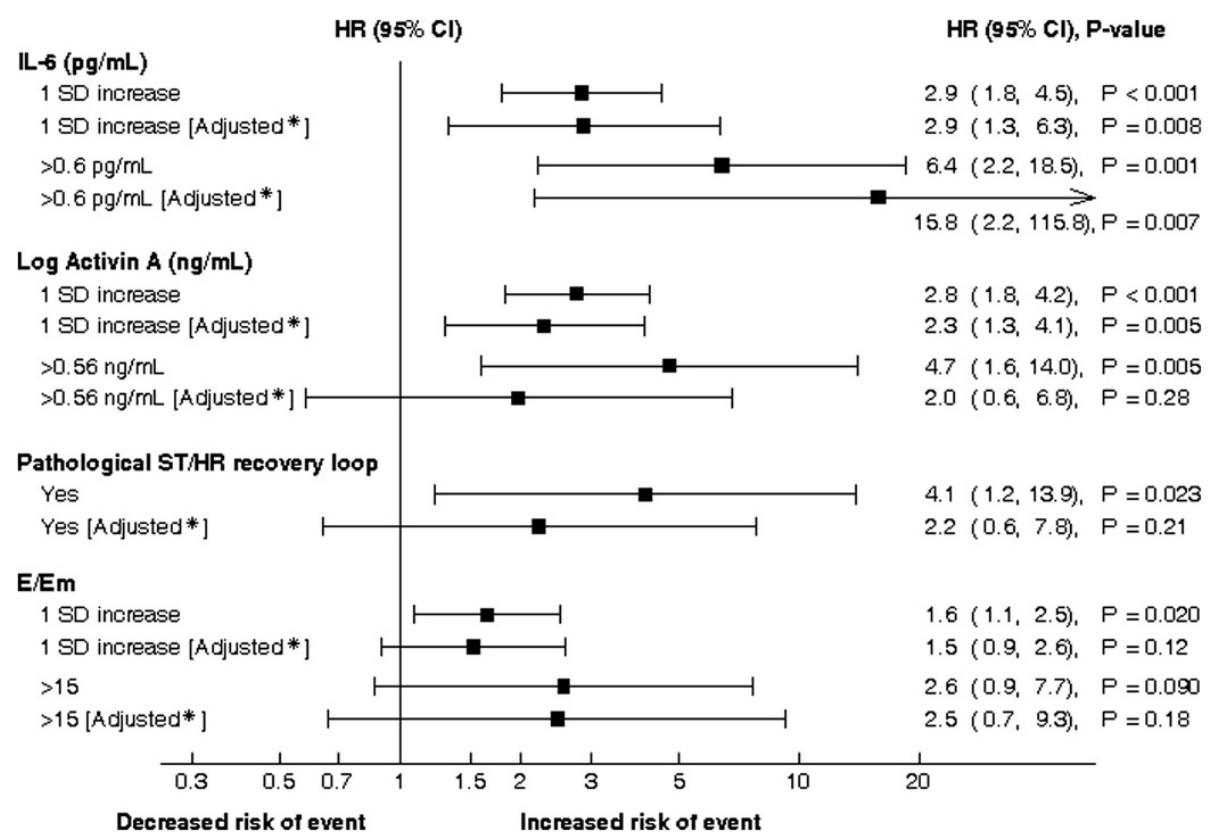

Figure 2 Unadjusted and adjusted hazard ratios for major CV events and death for the novel CV risk markers. * adjusted for age, gender, known CVD at inclusion, diastolic blood pressure, s-HDL-cholesterol, log microalbuminuria and s-creatinine. Abbreviations: CV= cardiovascular, IL-6 = Interleukin-6, SD = Standard Deviation.

CRP with CV events and death in this population with type 2 diabetes. The reason for this finding is at present not clear, but underscores that it is unlikely that one marker could reflect all inflammatory pathways involved in complex disorders like type 2 diabetes and its $\mathrm{CV}$ complications. Moreover, although IL-6 is an up-stream activator of CRP synthesis, this pleiotrophic cytokine also has a wide range of other effects not related to induction of acute phase proteins.

$\mathrm{E} / \mathrm{Em}$ is a reliable predictor of major CV events and death in chronic renal failure, ischemic heart disease and in hypertrophic cardiomyopathy $[8,9,38]$. Our data indicated that $\mathrm{E} / \mathrm{Em}$, an easily attainable ratio in those patients that undergo echocardiography, is associated with major

Table 2 C-statistics and net reclassification improvement (NRI) for the conventional risk markers alone, and in combination with one or more novel risk marker

\begin{tabular}{lll}
\hline & Harrell's C & NRI, P-value \\
\hline Standard (STD) model alone & 0.794 & Reference model \\
STD + IL-6 & 0.913 & $43.2 \%, \mathrm{p}=0.001$ \\
STD + log ActivinA & 0.859 & $20.3 \%, \mathrm{p}=0.013$ \\
STD + IL-6 + log ActivinA & 0.923 & $46.5 \%, \mathrm{p}<0.001$ \\
STD + E/Em + pathol recovery loop & 0.891 & $21.5 \%, \mathrm{p}=0.099$
\end{tabular}

The NRI calculations are based on estimated probabilities of event from logistic regression models. The three risk categories used are defined by the probability cut-offs $5 \%$ and $10 \%$.

STD model: age, gender, known CVD, diastolic blood pressure, microalbuminuria and serum levels of HDL-cholesterol and creatinine Abbreviations: STD Standard, IL-6 Interleukin-6, pathol Pathological.
$\mathrm{CV}$ events and death also in people with type 2 diabetes. We did, however, not find a clear improvement in the prediction of outcome when adding $\mathrm{E} / \mathrm{Em}$ to a model consisting of conventional $\mathrm{CV}$ risk factors, potentially at least partly reflecting a relatively low number of patients.

Likewise, although we found that pathological recovery loop was significantly associated to outcome, it did not improve prediction of this beyond standard risk factor assessment.

\section{Limitations}

Our study has some limitations including a potential low generalizability since all patients were Caucasians and examined at the same center. The number of participants was relatively low and not all had IL-6 and E/Em measured, which led to an even more reduced number of events that could be related to these markers and tests. Our study is also insufficiently powered to analyze the individual components of the composite endpoint in fatal and non-fatal CV events. Moreover, less than 1/3 of the first events had cardiac origin; the remaining being strokes, stroke related deaths, or death from non CV causes.

The strength of our study is foremost the long observationtime and accurate follow-up data on outcome, and that few patients were lost to follow-up.

\section{Conclusion}

Circulating levels of IL-6 and activin A may provide prognostic information on $\mathrm{CV}$ events and mortality beyond 
traditional CV risk factors. As these both are easily implementable in clinical practice they could improve CV prevention strategies in people with type 2 diabetes. We suggest that these parameters as well as E/Em and pathological recovery loop should be further validated in other and larger cohorts of patients with type 2 diabetes.

\section{Abbreviations}

MACE: Major adverse cardiovascular event; CV: Cardiovascular:

CVD: Cardiovascular disease; CAD: Coronary artery disease; IL: Interleukin;

CPET: Cardiopulmonary exercise test; TDI: Tissue doppler imaging: MI: Myocardial infarction; UAP: Unstable angina pectoris; hsCRP: High sensitivity C-reactive protein; ST/HR: ST/heart rate; LV: Left ventricular; LVEF: Left ventricular ejection fraction; BMI: Body mass index; NRI: Net reclassification improvement.

\section{Competing interests}

Odd Erik Johansen is employed by Boehringer Ingelheim. Kåre I. Birkeland has served as a consultant for or received lecture fees or travel reimbursement from Novo Nordisk, Eli Lilly, Sanofi-Aventis, MSD, Boehringer Ingelheim, Bristol-Myers Squibb, Novartis or Pfizer.

Lars Gullestad has received lecture fees from Eli Lilly. All other authors declare no competing interests.

\section{Authors' contributions}

OEJ, KIB and LG. designed the study. APO, EO, OEJ, SA, KE, TU and PA collected and researched the data. APO coordinated the writing of the manuscript. MWF was the statistical expert. All authors contributed to discussion, reviewed the manuscript, and approved the final work. APO and OEJ are the guarantors of this work and, as such, had full access to all the data in the study and take responsibility for the integrity of the data and the accuracy of the data analysis.

\section{Acknowledgment}

We thank all the patients for devoting their time to participate. We also thank the staff of the Echocardiography unit at Oslo University Hospital Rikshospitalet, the staff of the Diabetes Outpatient Clinic and of the Department of Medical Research, Bærum Hospital, Vestre Viken Health Trust, for helpful assistance and cooperation. This work was supported financially by the South-Eastern Norway Regional Health Authority, Vestre Viken Hospital Trust, the Norwegian Diabetes Association, and the Johan Selmer Kvanes' Endowment.

\section{Author details}

'Department of Medical Research, Bærum Hospital, Vestre Viken Hospital Trust, N-1309 Rud, Bærum, Norway. ${ }^{2}$ Department of Cardiology, Oslo University Hospital Rikshospitalet, Oslo, Norway. ${ }^{3}$ K.G.Jebsen Cardiac Research Centre and Center for Heart Failure Research, Faculty of Medicine, University of Oslo, Oslo, Norway. ${ }^{4}$ Research Institute of Internal Medicine, Oslo University Hospital Rikshospitalet, Oslo, Norway. ${ }^{5}$ Section of Clinical Immunology and Infectious Diseases, Oslo University Hospital Rikshospitalet, Oslo, Norway. ${ }^{6}$ Institute of Clinical Medicine, University of Oslo, Oslo, Norway. ${ }^{7}$ Unit of Biostatistics and Epidemiology, Oslo University Hospital, Oslo, Norway. ${ }^{8}$ Department of Endocrinology, Obesity and Preventive medicine, Oslo University Hospital Aker, Oslo, Norway.

Received: 4 July 2013 Accepted: 26 August 2013

Published: 30 August 2013

\section{References}

1. Vaccaro O, Eberly LE, Neaton JD, Yang L, Riccardi G, Stamler J: Impact of diabetes and previous myocardial infarction on long-term survival: 25-year mortality follow-up of primary screenees of the Multiple Risk Factor Intervention Trial. Arch Intern Med 2004, 164(13):1438-1443.

2. Sloan FA, Bethel MA, Ruiz D Jr, Shea AM, Feinglos MN: The growing burden of diabetes mellitus in the US elderly population. Arch Intern Med 2008, 168(2):192-199. discussion 199.

3. Lee DP, Fearon WF, Froelicher VF: Clinical utility of the exercise ECG in patients with diabetes and chest pain. Chest 2001, 119(5):1576-1581.
4. Kligfield P, Ameisen O, Okin PM: Heart rate adjustment of ST segment depression for improved detection of coronary artery disease. Circulation 1989, 79(2):245-255.

5. Kligfield P: Principles of simple heart rate adjustment of ST segment depression during exercise electrocardiography. Cardiol J 2008, 15(2):194-200.

6. Johansen OE, Bjuro T, Endresen K, Blaasaas KG, Birkeland K, Aakhus S, Gullestad L Heart rate adjustments and analysis of recovery patterns of ST-segment depression in type 2 diabetes. Int J Cardiol 2008, 127(1):129-132.

7. Ommen SR, Nishimura RA, Appleton CP, Miller FA, Oh JK, Redfield MM, Tajik AJ: Clinical utility of Doppler echocardiography and tissue Doppler imaging in the estimation of left ventricular filling pressures: A comparative simultaneous Doppler-catheterization study. Circulation 2000, 102(15):1788-1794

8. Wang AY, Wang M, Lam CW, Chan IH, Zhang Y, Sanderson JE: Left ventricular filling pressure by Doppler echocardiography in patients with end-stage renal disease. Hypertension 2008, 52(1):107-114.

9. Hillis GS, Moller JE, Pellikka PA, Gersh BJ, Wright RS, Ommen SR, Reeder GS, Oh JK: Noninvasive estimation of left ventricular filling pressure by $E / \mathrm{e}^{\prime}$ is a powerful predictor of survival after acute myocardial infarction. $J$ Am Coll Cardiol 2004, 43(3):360-367.

10. Kitaoka H, Kubo T, Hayashi K, Yamasaki N, Matsumura Y, Furuno T, Doi YL: Tissue Doppler imaging and prognosis in asymptomatic or mildly symptomatic patients with hypertrophic cardiomyopathy. Eur Heart J Cardiovasc Imaging 2013, 14(6):544-549.

11. From AM, Scott CG, Chen HH: The development of heart failure in patients with diabetes mellitus and pre-clinical diastolic dysfunction a population-based study. J Am Coll Cardiol 2010, 55(4):300-305.

12. Phillips DJ, de Kretser DM, Hedger MP: Activin and related proteins in inflammation: not just interested bystanders. Cytokine Growth Factor Rev 2009, 20(2):153-164.

13. Naka T, Nishimoto N, Kishimoto T: The paradigm of IL-6: from basic science to medicine. Arthritis Res 2002, 4(Suppl 3):S233-242.

14. Danesh J, Kaptoge S, Mann AG, Sarwar N, Wood A, Angleman SB, Wensley F, Higgins JP, Lennon L, Eiriksdottir G, et al: Long-term interleukin-6 levels and subsequent risk of coronary heart disease: two new prospective studies and a systematic review. PLoS Med 2008, 5(4):e78.

15. Hingorani AD, Casas JP: The interleukin-6 receptor as a target for prevention of coronary heart disease: a mendelian randomisation analysis. Lancet 2012, 379(9822):1214-1224.

16. Johansen OE, Birkeland Kl, Orvik E, Flesland O, Wergeland R, Ueland T, Smith C, Endresen $\mathrm{K}$, Aukrust P, Gullestad L: Inflammation and coronary angiography in asymptomatic type 2 diabetic subjects. Scand I Clin Lab Invest 2007, 67(3):306-316

17. Ueland T, Aukrust P, Aakhus S, Smith C, Endresen K, Birkeland Kl, Gullestad L, Johansen OE: Activin A and cardiovascular disease in type 2 diabetes mellitus. Diab Vasc Dis Res 2012, 9(3):234-237.

18. Executive summary: standards of medical care in diabetes-2013. Diabetes Care 2013, 36(Supplement 1):S4-S10.

19. Gottdiener JS, Bednarz J, Devereux R, Gardin J, Klein A, Manning WJ, Morehead A, Kitzman D, Oh J, Quinones M, et al: American Society of Echocardiography recommendations for use of echocardiography in clinical trials. J Am Soc Echocardiogr 2004, 17(10):1086-1119.

20. Lang RM, Bierig M, Devereux RB, Flachskampf FA, Foster E, Pellikka PA, Picard MH, Roman MJ, Seward J, Shanewise JS, et al: Recommendations for chamber quantification: a report from the American Society of Echocardiography's Guidelines and Standards Committee and the Chamber Quantification Writing Group, developed in conjunction with the European Association of Echocardiography, a branch of the European Society of Cardiology. J Am Soc Echocardiogr 2005, 18(12):1440-1463.

21. Gaede P, Lund-Andersen H, Parving HH, Pedersen O: Effect of a multifactorial intervention on mortality in type 2 diabetes. N Engl J Med 2008, 358(6):580-591.

22. Lydersen S, Fagerland MW, Laake P: Recommended tests for association in $2 \times 2$ tables. Stat Med 2009, 28(7):1159-1175.

23. Pencina MJ, D'Agostino RB Sr, D'Agostino RB Jr, Vasan RS: Evaluating the added predictive ability of a new marker: from area under the ROC curve to reclassification and beyond. Stat Med 2008, 27(2):157-172. discussion 207-112.

24. Spranger J, Kroke A, Mohlig M, Hoffmann K, Bergmann MM, Ristow M, Boeing $H$, Pfeiffer AF: Inflammatory cytokines and the risk to develop type 2 diabetes: results of the prospective population-based European 
Prospective Investigation into Cancer and Nutrition (EPIC)-Potsdam Study. Diabetes 2003, 52(3):812-817.

25. Koenig W, Khuseyinova N: Biomarkers of atherosclerotic plaque instability and rupture. Arterioscler Thromb Vasc Biol 2007, 27(1):15-26.

26. Sarwar N, Butterworth AS, Freitag DF, Gregson J, Willeit P, Gorman DN, Gao P, Saleheen D, Rendon A, Nelson CP, et al: Interleukin-6 receptor pathways in coronary heart disease: a collaborative meta-analysis of 82 studies. Lancet 2012, 379(9822):1205-1213.

27. Martin-Cordero L, Garcia JJ, Hinchado MD, Ortega E: The interleukin-6 and noradrenaline mediated inflammation-stress feedback mechanism is dysregulated in metabolic syndrome: effect of exercise. Cardiovasc Diabetol 2011, 10:42.

28. Zhou Y, Wei Y, Wang L, Wang X, Du X, Sun Z, Dong N, Chen X: Decreased adiponectin and increased inflammation expression in epicardial adipose tissue in coronary artery disease. Cardiovasc Diabetol 2011, 10(1):2.

29. Ridker PM, Hennekens $\mathrm{CH}$, Buring JE, Rifai N: C-reactive protein and other markers of inflammation in the prediction of cardiovascular disease in women. N Engl J Med 2000, 342(12):836-843.

30. Ridker PM, Rifai N, Stampfer MJ, Hennekens CH: Plasma concentration of interleukin- 6 and the risk of future myocardial infarction among apparently healthy men. Circulation 2000, 101(15):1767-1772.

31. Harris TB, Ferrucci L, Tracy RP, Corti MC, Wacholder S, Ettinger WH Jr Heimovitz H, Cohen HJ, Wallace R: Associations of elevated interleukin-6 and C-reactive protein levels with mortality in the elderly. Am J Med 1999, 106(5):506-512.

32. Davenport C, Ashley DT, O'Sullivan EP, Corley BT, Fitzgerald P, Agha A, Thompson CJ, O'Gorman DJ, Smith D: Identifying coronary artery disease in men with type 2 diabetes: osteoprotegerin, pulse wave velocity, and other biomarkers of cardiovascular risk. J Hypertens 2011, 29(12):2469-2475.

33. Herder C, Schottker B, Rothenbacher D, Roden M, Kolb H, Muller H, Brenner H Interleukin-6 in the prediction of primary cardiovascular events in diabetes patients: results from the ESTHER study. Atherosclerosis 2011, 216(1):244-247.

34. Sideras P, Apostolou E, Stavropoulos A, Sountoulidis A, Gavriil A, Apostolidou A, Andreakos E: Activin, neutrophils, and inflammation: just coincidence? Semin Immunopathol 2013, 35(4):481-99.

35. Andersen GO, Ueland T, Knudsen EC, Scholz H, Yndestad A, Sahraoui A, Smith C, Lekva T, Otterdal K, Halvorsen B, et al: Activin A levels are associated with abnormal glucose regulation in patients with myocardial infarction: potential counteracting effects of activin A on inflammation. Diabetes 2011, 60(5):1544-1551.

36. Wu H, Wu M, Chen Y, Allan CA, Phillips DJ, Hedger MP: Correlation between Blood Activin Levels and Clinical Parameters of Type 2 Diabetes. Exp Diabetes Res 2012, 2012:410579.

37. Weigert J, Neumeier M, Wanninger J, Schober F, Sporrer D, Weber M, Schramm A, Wurm S, Stogbauer F, Filarsky M, et al: Adiponectin upregulates monocytic activin A but systemic levels are not altered in obesity or type 2 diabetes. Cytokine 2009, 45(2):86-91.

38. Kitaoka H, Kubo T, Hayashi K, Yamasaki N, Matsumura Y, Furuno T, Doi YL: Tissue Doppler imaging and prognosis in asymptomatic or mildly symptomatic patients with hypertrophic cardiomyopathy. Eur Heart J Cardiovasc Imaging 2012, 14(6):544-9.

doi:10.1186/1475-2840-12-126

Cite this article as: Ofstad et al:: Interleukin- 6 and activin A are independently associated with cardiovascular events and mortality in type 2 diabetes: the prospective Asker and Bærum Cardiovascular Diabetes (ABCD) cohort study. Cardiovascular Diabetology 2013 12:126.

\section{Submit your next manuscript to BioMed Central and take full advantage of:}

- Convenient online submission

- Thorough peer review

- No space constraints or color figure charges

- Immediate publication on acceptance

- Inclusion in PubMed, CAS, Scopus and Google Scholar

- Research which is freely available for redistribution 